\title{
Event-related potentials elicited by spoken relative clauses
}

\author{
Horst M. Müller a, * Jonathan W. King a, Marta Kutas a,b \\ ${ }^{a}$ Department of Cognitive Science, University of California at San Diego, 9500 Gilman Drive, La Jolla, CA 92093-0515, USA \\ b Department of Neurosciences, University of California at San Diego, 9500 Gilman Drive, La Jolla, CA 92093-0515, USA
}

Accepted 10 September 1996

\begin{abstract}
Sentence-length event-related potential (ERP) waveforms were obtained from 23 scalp sites as 24 subjects listened to normally spoken sentences of various syntactic structures. The critical materials consisted of 36 sentences each containing one of two types of relative clauses that differ in processing difficulty, namely Subject Object (SO) and Subject Subject (SS) relative clauses. Sentence-length ERPs showed several differences in the slow scalp potentials elicited by SO and SS sentences that were similar in their temporal dynamics to those elicited by the same stimuli in a word-by-word reading experiment, although the effects in the two modalities have non-identical distributions. Just as for written sentences, there was a large, fronto-central negativity beginning at the linguistically defined 'gap"' in the SO sentences; this effect was largest for listeners with above-median comprehension rates and is hypothesized to index changes in on-line processing demands during comprehension.
\end{abstract}

Keywords: Event-related potential (ERP); Auditory language processing; Relative clause; Slow brain potential; Working memory; Left anterior negativity (LAN); Individual difference

\section{Introduction}

Within the realm of cognitive abilities, the use of language is among the most impressive skills of the human animal, far outstripping those of other species and machines of our own creation. Most of what we know about how language is implemented in the brain comes from studies of derivative language processes like reading. This is the case even though reading and writing took untold tens of thousands of years to evolve even after our ancestors spoke and both of these skills require many years of training even after a child communicates perfectly via speech (e.g. [22]). Furthermore, the comprehension of written language is a multimodal process that involves neural activity in both the language and visual systems, while activity in the auditory system, although it is more closely related phylogenetically to language processing, becomes unnecessary. Therefore, while reading and listen-

\footnotetext{
* Corresponding author. Universität Bielefeld, Fakultät für Linguistik und Literaturwissenschaft, AG Experimentelle Neurolinguistik, Postfach 100 131, 33501 Bielefeld, Germany. Fax: + 49 (521) 106-2996; E-mail: mueller@cogsci.ucsd.edu
}

ing undoubtedly share features in common, they are clearly not identical. In this report we describe an investigation of on-line auditory sentence processing using the event-related potentials (ERPs) of the brain as our dependent measure as a first step in better defining the processes of speech comprehension in particular and language comprehension in general.

One way to investigate a facet of language comprehension is by comparing the effects elicited by processing stimulus types that are as close as possible to being "minimal pairs" in terms of their phonological, syntactic, and semantic-pragmatic features. Miller and Chomsky [21] were among the first to notice that there were English sentences whose syntactic structures were quite similar (at least by then-current theories), but whose processing demands were not, as in the following examples:

(SS) The fireman who speedily rescued the cop sued the city over working conditions.

(SO) The fireman who the cop speedily rescued sued the city over working conditions.

While both sentences contain embedded relative clauses that modify the same noun phrase (NP; in our example, the fireman), most readers probably share the intuition that the SO sentence is more difficult to understand, despite the 
fact that both sentence types are utterly unambiguous ${ }^{1}$. Why this is so has been the subject of much research, and many factors appear to contribute. Historically, one of the more prominently mentioned factors is that structures like the SO sentence induce a greater working memory load. One argument for this working memory load hypothesis is that the syntactic processing of the initial NP in the SO sentence (e.g., the fireman) is interrupted by the need to process an (self-)embedded NP (the cop) which requires the temporary storage of the sentence initial NP in working memory until its processing can resume (see, e.g. [21]).

The great appeal of this proposal was that it appeared to correctly predict the increasing difficulty of processing SO relative to SS sentences as clauses become more deeply embedded; this is why a sentence like The fireman who the cop who the doctor speedily cured rescued sued the city is essentially impossible to comprehend on first reading, and certainly more difficult than a comparable SS sentence such as The fireman who speedily rescued the doctor who cured the cop sued the city. One difficulty with this kind of purely "interrupt-and-store" account, however, is that singly embedded SO sentences are also quite difficult to comprehend (see, e.g. [11]) even though the working memory load, characterized in terms of the quantity of storage required, does not seem excessive. Another difficulty with such theories is that they tend to predict one locus of processing difficulty - at the point where the NP is "saved away" during the interruption - that does not correspond to the sentence location where reaction time measures of processing difficulty are actually observed (see, e.g. [5]). A somewhat different version of the storage hypothesis was later detailed by Wanner and Maratsos [36], who suggested that the more crucial factor in processing singly embedded SO sentences was the distance or time over which the initial NP was maintained and the required "reactivation" of this NP from the storage buffer when it was needed at a future point in the syntactic analysis.

In contrast to these storage-oriented views are theories that emphasize differences in the kind of processing required to comprehend SO and SS sentences. One source of additional processing may be that the grammatical role played by the head noun phrase changes in an SO sentence but is constant in an SS sentence (e.g. [36]). Thus, in an SO sentence the head noun serves as the grammatical subject of the main clause, but as the object of the embedded clause, while in an SS sentence the head noun has the same grammatical role in the two clauses. Further, when the verbs used in these two sentences are active and

\footnotetext{
${ }^{1}$ To clarify the terminology, the first word in the terms "subject object relative" and "subject subject relative" refers to the fact that it is the subject noun phrase (NP) of the sentence that is being relativized, while the second word specifies whether the main clause subject NP corresponds to the object or the subject of the embedded relative clause.
}

strongly transitive, the thematic roles played by the two noun phrases mirror the grammatical roles in differing between the two sentence types. This factor might be especially salient in a language like English which essentially lacks case or role marking and relies on a combination of word order to provide grammatical case information and the thematic role preferences of verbs to assign thematic roles to discourse participants. This is even more true of contemporary American dialects where the distinction between nominative vs. accusative case marking on the relative pronoun (i.e., who vs. whom) has fallen into disuse.

Similar contrasts in processing difficulty likewise might be attributed to the process of constructing referential representations on-line, given that the SO shift of thematic role also correlates with a shift in the identity of the participant whose perspective is believed to be mirrored by the reader or listener. Thus, MacWhinney and Pleh [18] have shown that much of the difference in processing $\mathrm{SO}$ and SS structures could be accounted for by manipulating word order systematically in Hungarian relative clauses; although case-marking was always unambiguous, word orders and clause types that required perspective shifts were more difficult to process than those which did not.

ERP measures provide a means for assessing the importance of these and other dynamic theories in that they can be recorded throughout the course of processing whole sentences and examined to determine at what points in the sentence the processing differs, and what the character of the difference is. This is in contrast to chronometric data, which have proven useful in studying the time course of these processes, but do not always afford unambiguous inferences from latency to specific processes. Thus, while one might expect to observe some reading time correlate of the maintenance of a working memory load during the processing of the SO relative clause, one cannot predict its direction. That is, one could predict slower times if latency follows difficulty as is usually assumed, or faster times if maintaining the memory load is taxing enough to cause a processing speed-up (i.e., speeding up to get to the verb before decay affects the contents of the NP memory trace).

Reaction time evidence for increased processing difficulty in SO sentences, nonetheless, has been found in numerous experiments that have examined gaze durations (e.g., with analogous French sentences [8]), continuous lexical decisions (e.g. [5]), and word-by-word reading times (e.g. [11,33]). All these latency measures are in accord: English SO sentences are more difficult to process, and the locus of the processing time increase occurs at and just following the end of the relative clause (i.e., at and just after the "gap" in the relative clause).

These findings are reasonable given either some version of the reactivation hypothesis or most of the processing hypotheses. In addition, these findings also emphasize the possibility that the locus of the difficulty is at the main clause verb because multiple thematic role assignments 
must be completed here or at some point just following (although the process of the thematic role assignment may begin earlier; see [37]). As the discourse participant referred to by the subject NP turns out to play different roles in the relative and main clauses, this may well have an additional effect on reading times for the main clause verb; indeed, this is the sentence location where the largest reading time effects are typically found.

As cognitive neuroscientists, we further recognize that behavioral measures alone cannot go far towards identifying the neural substrates of these processes, and proving whether they are similar or different across modalities. In our laboratory, we have begun to address these and other issues involved in sentence processing using measures based on scalp-recorded event-related brain potentials (ERPs; reviewed in, e.g. [16]). Recent work has shown that at least one component of the ERP, a left-lateralized anterior negativity (LAN) appearing about $300 \mathrm{~ms}$ post word onset differentiates the ERPs to (function) words that directly followed the syntactic "gap" in a sentence when compared to control words where there was no gap $[13,14]$. Kluender and Kutas [14] suggested that this LAN effect might be an index of the working memory operations involved.

King and Kutas [12] have investigated the visual processing of the particular sentence types discussed in this paper during word-by-word reading In that study, a LAN effect was elicited by a content word (e.g., a verb) following the gap in the relative, namely the main clause verb in the $\mathrm{SO}$ relative to $\mathrm{SS}$ sentences. Because $\mathrm{SO}$ and $\mathrm{SS}$ sentences are well controlled both for length and identity of the lexical items involved, they could be compared electrophysiologically not only on a word-by-word basis but across their entire extent. The latter analysis revealed a substantial negativity for SO (relative to SS) sentences that became noticeable from the start of the relative clause continuing through the processing of the main clause. This slow brain potential effect was similar to that previously reported for other kinds of complex processing tasks that presumably made greater than normal demands on work-

(SS) The barber who patiently loved the waitress examined the ring studded with diamonds.

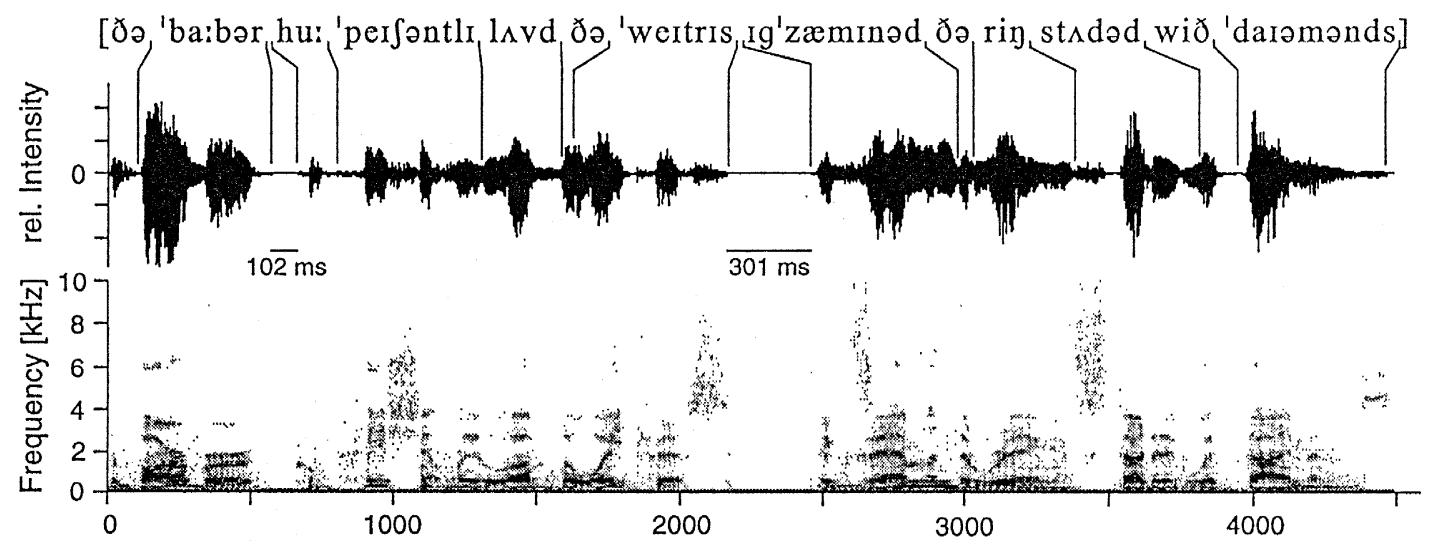

(SO) The fireman who the cop speedily rescued sued the city over working conditions.

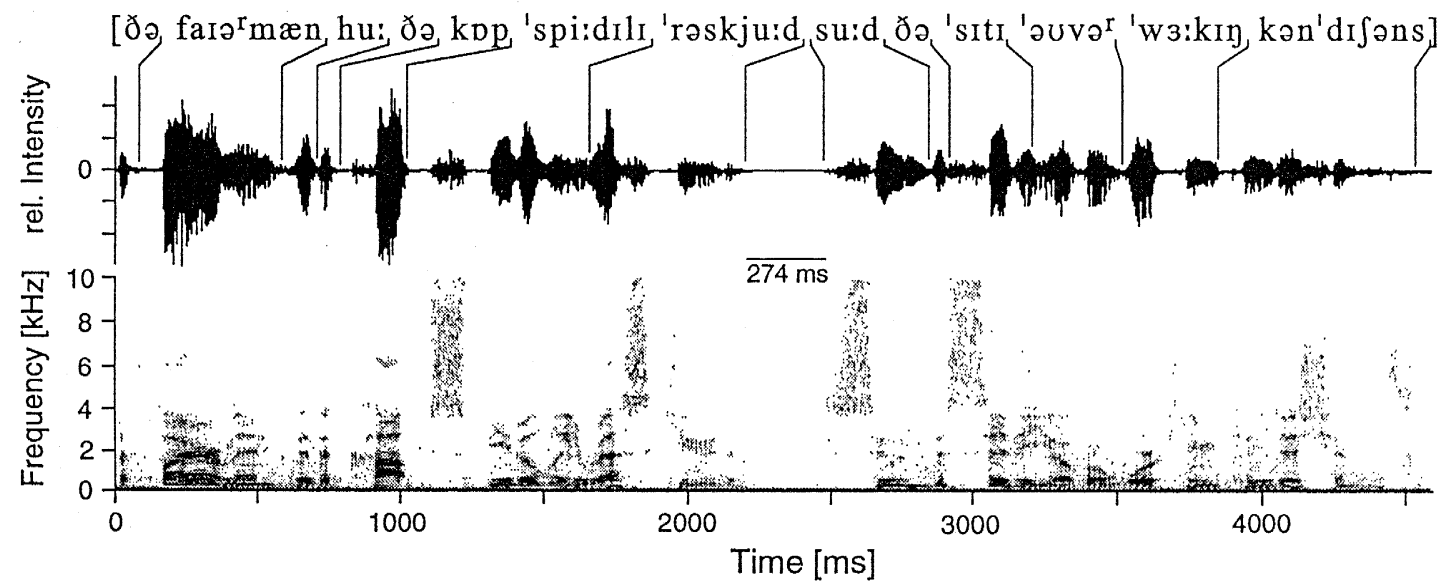

Fig. 1. Spectrograms of sample stimuli in the intensity and frequency domain with accompanying phonetic transcriptions of two critical sentences used in this experiment, with the top panel depicting a standard SS sentence and the bottom panel depicting an SO sentence. 
Table 1

Critical time regions of SS and SO sentences used

\begin{tabular}{lll}
\hline Pre-relative clause & Relative clause & Gap \\
\hline (SS) The fireman who & speedily rescued the cop & sued the city over working... \\
(SO) The fireman who & the cop speedily rescued & sued the city over working... \\
$0-700 \mathrm{~ms}$ & $700-2300 \mathrm{~ms}$ & $2600-4000 \mathrm{~ms}$ \\
\hline
\end{tabular}

ing memory (as reviewed by, e.g. [29,31]). Its size was modulated by the comprehension skill of the individuals, being largest in better comprehenders and quite small in poor comprehenders. While this work represented a substantial advance over what could be inferred from reading time data alone, there remained some questions about whether the ERP components recorded were indexing processes specific to reading or language. In addition, there were other methodological questions about the ecological validity of reading sentences one word at a time, at the rate of one word every $500 \mathrm{~ms}$. Recent work, however, has established the feasibility of doing ERP studies using auditorily presented materials (e.g. $[3,4,7,24,39]$ ). These pioneering efforts encouraged us to conduct the current experiment on the processing of SO and SS sentences presented as natural speech.

\section{Materials and methods}

\subsection{Subjects}

Twenty-four participants (12 women) received course credit or $\$ 5.00 / \mathrm{h}$ to participate in sessions that lasted approximately $3 \mathrm{~h}$. All participants were right-handed native English speakers between the ages of 18 and 35 (mean: 23.3) without any known neurological problems and had normal hearing thresholds (and no threshold asymmetries greater than $3 \mathrm{~dB}$ SPL) as assessed by pure tone audiometry. Of the 24 participants, 9 had first order family members who were left-handed.

\subsection{Stimuli}

All 216 sentences in the experiment were syntactically and semantically congruent English sentences spoken by a trained, male speaker with natural intonation and at a normal rate. Mean stimulus intensity ranged between 50 and $55 \mathrm{~dB}$ SPL, which corresponds to the loudness of a quiet conversation. The 72 critical items consisted of 36 SO- and 36 SS-relative sentences pseudo-randomly dispersed among 144 "'filler" sentences. The mean duration of the critical sentences averaged 4545 ms (range: $3743-$ $5403 \mathrm{~ms}$ ) for the SO sentences and $4672 \mathrm{~ms}$ (3797-5659 $\mathrm{ms})$ for the SS sentences. Annotated spectrograms of actual stimuli of both types appear in Fig. 1. To perform the multiple clause analyses presented here, we averaged 5000 $\mathrm{ms}$ epochs including a $500 \mathrm{~ms}$ pre-stimulus baseline, time- locked to the beginning of the sentence-initial words and decimated (down-sampled) to $125 \mathrm{~Hz}$.

For the purposes of statistical analysis, mean amplitude measurements were taken within non-overlapping latency windows whose borders were chosen to fall at what were, on average, the linguistic constituent boundaries of interest in the two sentence types. Thus, the sentences were divided into the pre-relative clause region $(0-700 \mathrm{~ms}$ postsentence onset), the relative-clause region $(700-2300 \mathrm{~ms}$ post onset), and the post relative-clause region (2300-4000 $\mathrm{ms}$ ). The relative clause region, in turn, was divided into early $(700-1500 \mathrm{~ms})$ and late $(1500-2300 \mathrm{~ms})$ subregions, while the post relative-clause region was divided into regions corresponding to the immediate post-gap area (2300-2600 ms), the main-verb area (2600-3000 ms) and the main-clause resolution area $(3000-4000 \mathrm{~ms})$. These regions are further illustrated in Table 1.

\subsection{Procedure}

Participants were asked to listen to the sentences in order to answer auditorily presented comprehension probes, which followed $38 \%$ of the sentences. The probes were simple TRUE/FALSE comprehension items that directly queried comprehension of the immediately preceding sentence (see [12], for details). Comprehension items were preceded by a warning tone that occurred $500 \mathrm{~ms}$ previous to it. A median split on comprehension performance was used to define "good" and "poor" comprehension groups.

\subsection{ERP recording}

Participants' electroencephalograms (EEGs) were recorded from a total of 23 scalp electrodes embedded in an electrocap and 2 electrodes affixed to the mastoids bilaterally. These sites included 11 from the 10-20 system (F7, F8, F3, F4, T5, T6, O1, O2, Fz, Cz, Pz) and 3 pairs of sites that were nominally over Broca's area and Wernicke's area and their right hemisphere homologues, and auditory cortex bilaterally ${ }^{2}$. The horizontal electro-oculogram was recorded from electrodes placed at the outer canthi. Electrodes also were placed supra-orbitally under each eye in

\footnotetext{
${ }^{2}$ Broca's area sites were located at the midpoint of the line between F7(8) and T3(4), Wernicke's left and right sites were placed $30 \%$ of the inter-aural distance laterally as measured from the midline point that was $13 \%$ of the nasion-inion distance posterior to $\mathrm{Cz}$, auditory cortex sites were $33 \%$ of the inter-aural distance lateral to $\mathrm{Cz}$.
} 
order to detect trials that included eye movements or blinks. For the whole sentence data reported here, about $30 \%$ of the trials were rejected due to artifacts.

All sites were referenced to a balanced non-cephalic recording whose independent leads were placed at the sterno-clavicular junction and on top of the seventh cervical vertebrae. The EEG was analog-filtered at acquisition between 0.01 and $100 \mathrm{~Hz}(\mathrm{TC} \cong 8.7 \mathrm{sec})$ and with a 60 $\mathrm{Hz}$ notch filter to attenuate power line noise. The data were digitally sampled at $250 \mathrm{~Hz}$ and stored on disk for subsequent analyses.

\section{Results}

\subsection{Behavioral results}

Overall comprehension accuracy was $77 \%$, which is comparable with previous work using these materials (e.g.
[12]). Comprehension accuracy for good comprehenders was $86 \%$ while poor comprehenders only managed $68 \%$ accuracy.

\subsection{Sentence-level ERP results}

The data from both good and poor comprehenders, shown in Fig. 2, indicate that sentence type had a large effect on the patterns of slow brain potentials. As expected from the reading data, SO sentences elicited a widespread negativity in comparison to the easier SS sentences. This difference begins in the relative-clause region (separated from preceding and following regions by dashed lines in Fig. 2), but becomes noticeably larger after the gap in the relative clause has been encountered. At the end of the relative clause $(\approx 2500 \mathrm{~ms}$ ), the ERP elicited by the SS sentences shows a phasic negativity that contrasts with the more prolonged negativity seen in SO sentences. More-
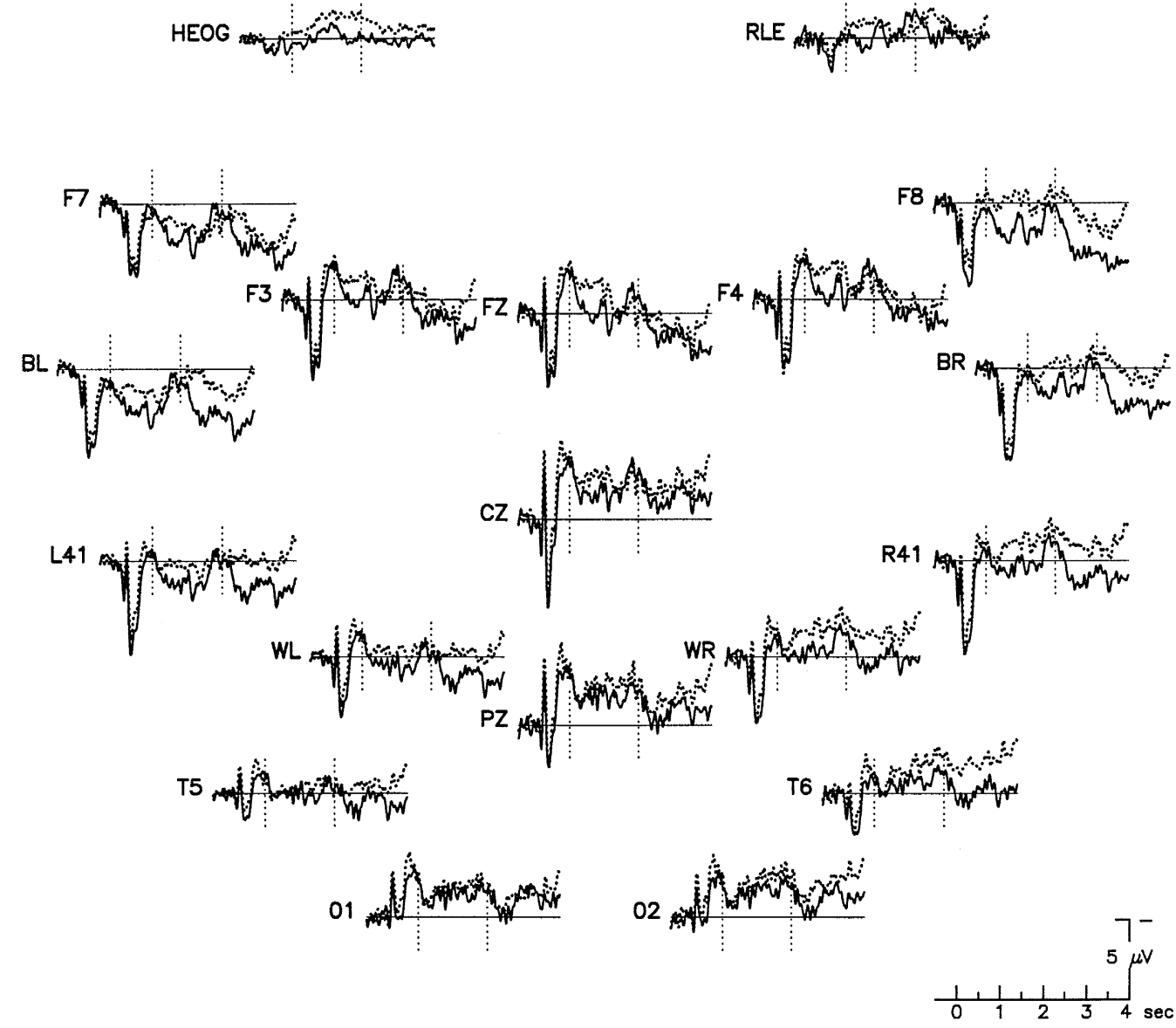

PRE-RELATIVE CLAUSE

RELATIVE CLAUSE

POST RELATIVE CLAUSE

\section{SS: The fireman who SO: The fireman who}

speedily rescued the cop

the cop speedily rescued

Fig. 2. Multiple-clause ERPs for SO and SS sentence types for all subjects $(n=24)$, with dotted vertical lines marking the temporal boundaries of the embedded relative clause. Note that the ERPs to SO sentences show a widespread negativity relative to the ERPs to SS sentences, especially over central sites bilaterally and much of the right hemisphere. 


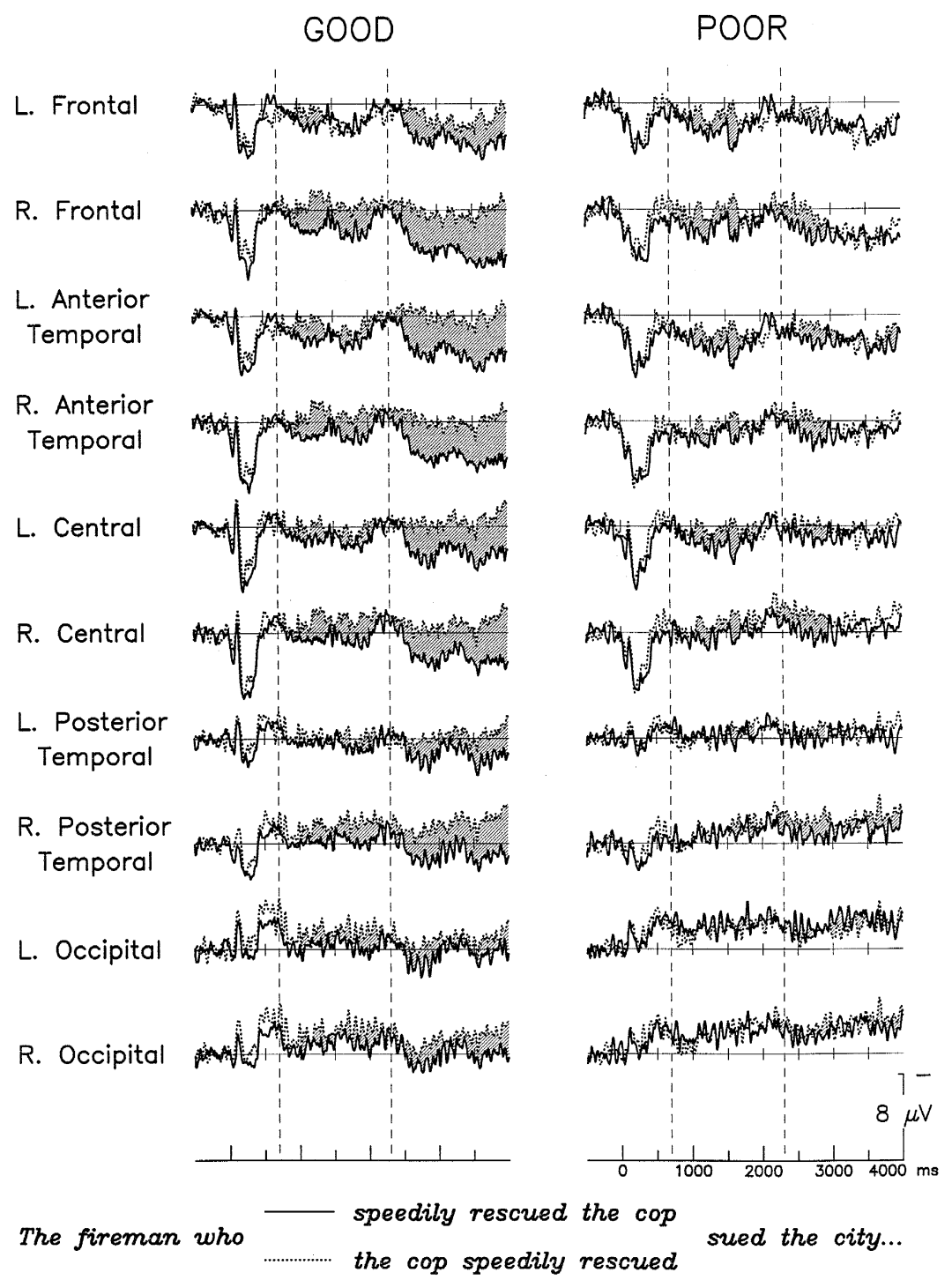

Fig. 3. Multiple-clause ERPs for SO and SS sentences at lateral sites for good $(n=12)$ and poor $(n=12)$ comprehenders, with dashed vertical lines marking the temporal boundaries of the embedded relative clause. Good comprehenders evince a much larger effect of sentence type than do poor comprehenders; this difference might be characterized as a greater positivity for SS sentences in good comprehenders.

over, whereas the ERP for the SS sentences shows a large positivity following this point, the ERP for the SO sentences stays negative. While these differences are widespread, they are slightly larger at fronto-central and posterior temporal sites, especially over the right hemisphere. The general pattern seen here is remarkably similar to that seen for visual materials with the exception of its laterality (e.g. [12]).

The laterality and antero-posterior distribution of these effects are most easily examined by restricting the analysis to the most lateral sites [15]. Thus, data from the most lateral sites were submitted to an ANOVA whose withinsubjects factors included Sentence Type (SO or SS), Hemisphere (left or right), and Electrode (frontal, anterior temporal, central, posterior temporal, and occipital), and whose between-subjects factor was comprehension status (good vs. poor, as discussed above). Separate ANOVAs were conducted for each of the time windows described in Table 1; a summary of these results is provided in Table $2^{3}$.

In addition to the nearly ubiquitous effect of Hemisphere (right hemisphere more negative), the overall effect of Sentence Type is reliable in every sentence region, and every subregion except that between 3000 and $3500 \mathrm{~ms}$ post-sentence onset; this exception, however, is qualified by a reliable interaction of Sentence Type by Comprehension $\left(F_{1,22}=4.31, P<0.05\right)$. The ERPs for good and poor comprehenders at the lateral sites are plotted in Fig. 3. Data from good comprehenders showed a continuation of the sustained relative negativity to $\mathrm{SO}$ sentences at the end of the main clause (i.e., 3000-3500 ms post onset).

\footnotetext{
${ }^{3}$ All main effects of Electrodes, being ubiquitous and large, are omitted from this table and Table 3.
} 
Table 2

Summary of ANOVA results for the relative clause experiment

\begin{tabular}{lccl}
\hline Region & Hemisphere & Type & Hemisphere $\times$ Type \\
& $F_{1,22}$ & $F_{1,22}$ & $F_{1,22}$ \\
\hline Relative-clause region & $16.78^{* * *}$ & 3.45 & $4.39 *$ \\
$\quad$ Early relative clause & $4.37^{*}$ & $8.90^{* *}$ & 1.93 \\
Late relative clause & $23.13^{* * *}$ & 2.11 & $4.62^{*}$ \\
& & & \\
Post-gap region & $10.84^{* *}$ & 1.26 & $6.95 *$ \\
Main verb & $4.22^{*}$ & $6.35^{*}$ & 2.04 \\
& & & \\
Post main verb region & $5.73^{*}$ & $8.09^{*} *$ & 2.17 \\
Main verb NP & 3.96 & 3.63 & 2.40 \\
Post NP & $6.61 *$ & $10.89 * *$ & 1.71
\end{tabular}

${ }^{*} P<0.05 ;{ }^{* *} P<0.01 ;{ }^{* * *} P<0.001$.

This was not true for data from poor comprehenders, where there was if anything a relative positivity in the ERPs elicited by SO sentences. Such positivities have been noted in the responses to ungrammatical sentences or to ambiguous sentences whose structurally favored interpretation is ungrammatical (see, e.g. [24], for work with auditory sentences). Our stimuli, however, are both well formed and completely unambiguous, which thus complicates the proposed interpretation of this positive effect. The interactions of Type and Hemisphere in the late relative clause region indicate that the difference between sentence types is largest over right hemisphere sites, and this asymmetry continues into the immediately following gap region. In principle, informative comparisons could be made between our current results with subject- and object-relative clauses in English and work with these same structures in German, which can be constructed to be ambiguous until the final word of the clause (e.g. [20,34]). The Mecklinger et al. study [20], however, used visually presented materials and did not report slow brain potential data, which are the focus of our work.

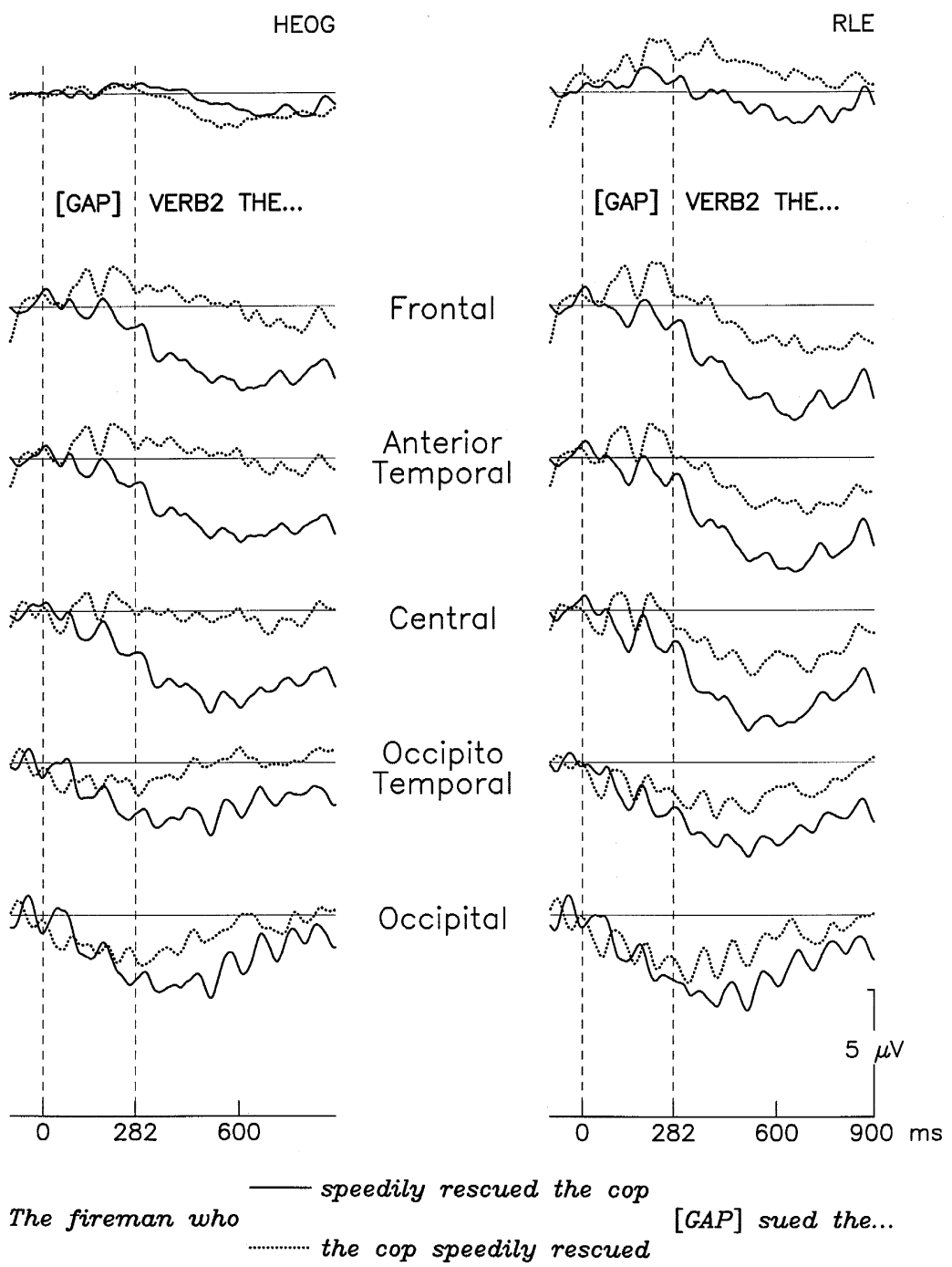

Fig. 4. Multiple-word ERPs elicited by the relative clause offset of SO and SS sentences for all subjects $(n=24)$ at lateral electrode sites, with dotted vertical lines indicating the beginning and average ending point of the post-clause silence previous to the main clause. Note that this silence is a phonetic gap for both SO and SS sentences but coincides with a syntactic gap for SO sentences only. 


\subsection{Single word analyses}

As can be seen from Figs. 2 and 3 and as has been noted elsewhere in this paper, words in a continuous sentence stream do not elicit very distinct ERPs. This is in part due to refractoriness of auditory ERP components, particularly the N1, and in part due to the fact that word boundaries are not themselves explicit in the speech signal (see, e.g., Fig. 1) but must be recovered during processing. Thus, while Fig. 2 shows that the first word of the sentence elicits a large and canonically distributed triphasic complex, this is not the case for other sentence positions, even in situations where the alignment of word onsets is reasonably exact. Thus, to a great extent the slow potential effects are the important data in a study of sentence processing like this one. The particular nature of our stimuli, however, makes it possible to investigate the evoked activity at one juncture which is also of great linguistic relevance, namely the onset of the silent period following the end of the relative clause in the two sentence types. As detailed in Section 2, the duration of this silence was equated between the two sentence types, usually by inserting additional silence at the end of the SS relative clause.

In both SS and SO sentences, the silence immediately follows the end of the relative clause, but only in the SO sentence is it essentially at the gap in the relative clause. Hence, one might infer that ERP differences between SS and SO sentences that begin at this point should reflect either the detection of the gap in the SO sentence, or the processing made necessary by the gap. Fig. 4 shows the grand mean waveforms recorded at lateral sites for the 900 ms epoch that begins at the acoustic end of the relative clause in both cases and ends in the middle of the main clause. Relative to the $100 \mathrm{~ms}$ pre-silence baseline, it can be seen that the ERP to the SO sentences is relatively more

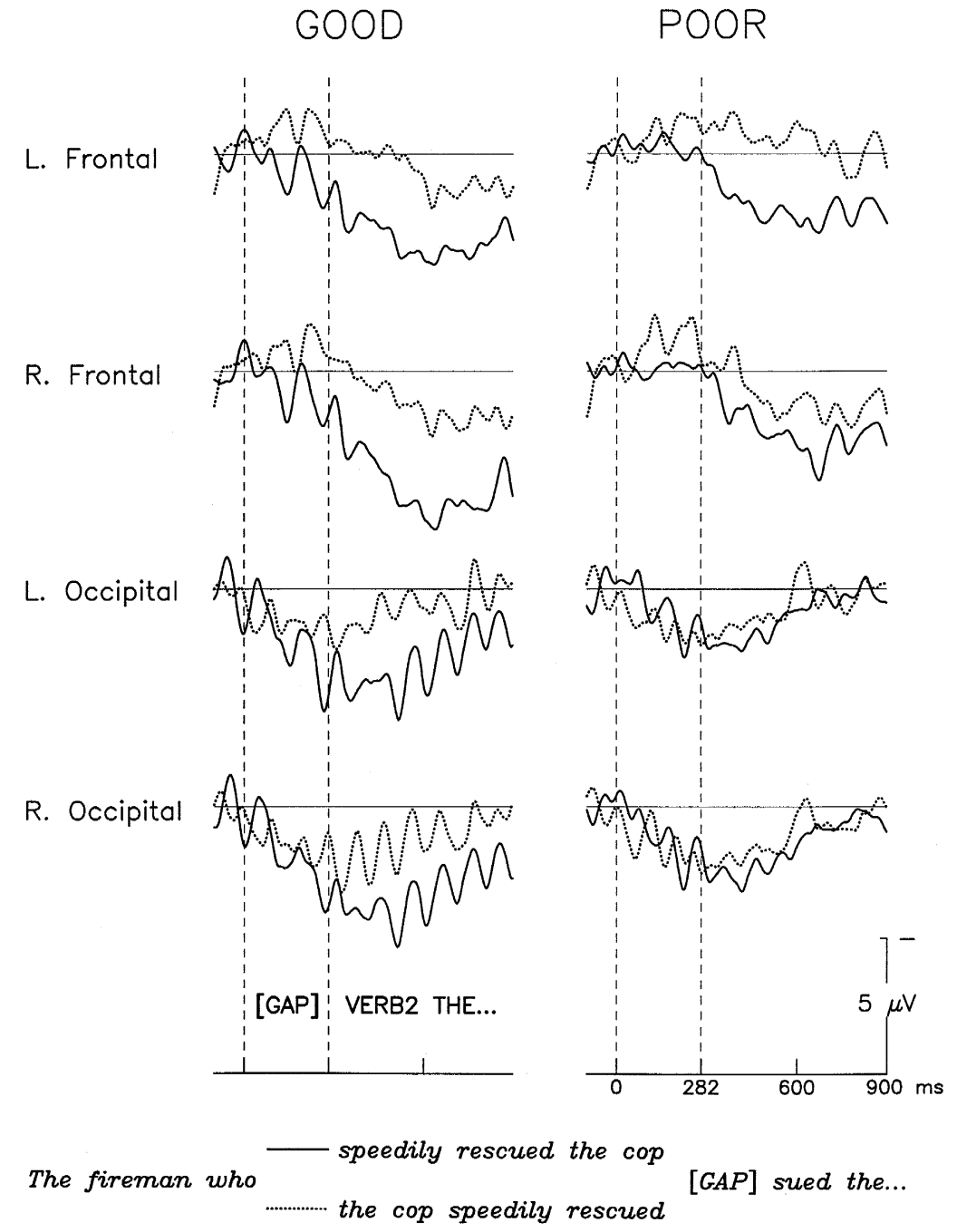

Fig. 5. Multiple-word ERPs elicited by relative clause offsets for good $(n=12)$ and poor $(n=12)$ comprehenders at frontal and occipital electrode sites, with dashed lines delineating the limits of the phonetic gap as in Fig. 4. 
Table 3

Summary of gap region ANOVA results

\begin{tabular}{rccll}
\hline Post onset & Type & Type $\times$ Electrode & Comprehension & $\mathrm{C} \times \mathrm{T} \times \mathrm{H} \times \mathrm{E}$ \\
& $F_{1,22}$ & $F_{1,22}$ & $F_{1,22}$ & $F_{4,88}$ \\
\hline $0-100$ & 0.51 & $4.00^{*}$ & 0.01 & 0.95 \\
$100-200$ & $2.30^{*}$ & $3.39^{+}$ & 2.15 & 0.68 \\
$200-300$ & $7.17^{* *}$ & $2.88^{+}$ & 2.01 & 0.55 \\
$300-400$ & $11.64^{* *}$ & $4.35^{*}$ & $4.67^{*}$ & 1.09 \\
$400-500$ & $15.85^{* *}$ & $2.96^{+}$ & $3.94^{+}$ & $2.58+$ \\
$500-600$ & $11.68^{* *}$ & $6.92^{*}$ & $7.42^{*}$ & $3.066^{+}$ \\
$600-700$ & $9.84^{* *}$ & $10.40^{* *}$ & $9.50^{* *}$ & $2.27^{+}$ \\
$700-800$ & $6.39^{*}$ & $5.17^{* *}$ & $5.94^{*}$ & 0.73 \\
$800-900$ & $6.17^{*}$ & $2.72^{+}$ & $9.15^{*}$ & 0.40 \\
\hline
\end{tabular}

${ }^{*} P<0.05 ;{ }^{*}{ }^{*} P<0.01 ;{ }^{* * *} P<0.001 ;{ }^{+} 0.05<P>0.10$.

negative than that to the SS sentences. The response to SS sentences appears to include a broadly distributed positivity following the end of the relative clause, perhaps reflecting the resolution of the immediately preceding clause-ending negativity (CEN; described in [15]).

Mean amplitude measures of the evoked activity at these sites were submitted to ANOVAs using the same factors as for the sentence ERP data. In order to assess the dynamics of processing, we looked at a series of nine 100 $\mathrm{ms}$ windows starting at the end of the relative clause (time 0 in Fig. 4) to a point $900 \mathrm{~ms}$ beyond that, approximately half-way through the main clause. Among the statistically reliable effects, we will concentrate on those main effects and interactions that included the factors of Sentence Type or Comprehension, shown in Table 3.

As suggested by Fig. 4, there is a reliable interaction of Sentence Type and Electrodes within the first $100 \mathrm{~ms}$ of the epoch, and a pervasive and reliable overall effect of sentence type from $200 \mathrm{~ms}$ after the end of the relative clause and beyond. This Sentence Type factor also reliably interacts with electrodes throughout most of the epoch, as the effect is larger over anterior sites (see Fig. 4). However, the lack of a 3-way interaction of Type, Hemisphere and Electrodes suggests that this is not strictly a LAN effect.

A natural question, given the effects of Comprehension Skill in the sentence-level analyses, is whether the ERPs of good and poor comprehenders also differ in this section of the sentence. Both the data plotted in Fig. 5 and the results given in Table 3 answer in the affirmative. In the second half of the epoch, in particular, the ERPs for both sentence types are more positive for the good than for the poor comprehenders. At the peak of this positivity between 400 and $600 \mathrm{~ms}$ post-gap onset the effect of Sentence Type appears to be restricted to left anterior electrodes for poor comprehenders but broadly and bilaterally distributed for good comprehenders. However, this pattern, as assessed by the significance of the 4-way Comprehension $\times$ Type $\times$ Hemisphere $\times$ Electrode interaction, is only marginally reliable after the Huynh-Feldt correction for violations of sphericity is applied. King and Kutas [12] observed a similar dissociation between the effects of Comprehension at more global versus local levels of analysis; that is, pointing towards a greater effect of Comprehension skill on slow brain potentials than on more transient evoked responses in sentence processing.

One aspect of these data that deserves some additional comment is the possibility that our adjustment of the pause duration for SS sentences may have had unforeseen consequences on the associated ERPs. In particular, our extension of this pause duration (to an average of $282 \mathrm{~ms}$ ) may have caused some subtle ERP effects, since the "expected" silence following a (non-gap) SS relative clause ending, while dependent on other phonological properties of the sentence, may be shorter on average than the silence following an SO relative clause ending. Thus, the prolongation of this pause in the SS condition may have on occasion elicited some missing stimulus activity in the ERP. Such effects have been found for longer pauses inserted in auditorily (but not visually) presented sentences (e.g. [2]), although the lower limit for such temporal disruption effects is not yet known.

\section{Discussion}

This work is an initial attempt to understand sentence processing mechanisms in natural speech, primarily by examining modulations in the slow brain potentials they elicit. Our success in finding reliable effects of syntactic structure that are modulated by a given reader's comprehension skill suggests that this technique might be much more widely applied. In particular, this method may be particularly appropriate for the study of language processing in various special populations, including the normal elderly and patients suffering from various forms of dementia or aphasia. While reaction time based methods have provided much valuable data about language processing in these populations, the inability of many subjects to generate movements that are fast and accurate enough to serve as on-line measurements of processing remains problematic. 
The slow brain potential effects we describe in this paper are temporally consistent with previous research done with visually presented sentences, showing greater negativity for the generally more demanding $\mathrm{SO}$ sentence type. The greater negativity for the SO relative to SS sentences began with the relative clause and extended to the sentences' ends, at least for the good comprehenders. Changes in the distribution of this negativity from predominantly frontal during the relative clause to somewhat more broadly distributed beyond the relative clause suggest that it comprises at least two different processes.

The effects in these speech data were somewhat different in their distribution from those we observed for wordby-word reading. Thus, while King and Kutas [12] showed a left hemisphere asymmetry for the effects of sentence type in some comparisons, in this study all effects are bilateral, or slightly larger over the right hemisphere. Although this study does not provide strong evidence for specific right hemisphere involvement in auditory sentence processing, one might be expected given its role in the processing of global prosodic cues that might be used to detect clause boundaries (e.g. [35]). It must be emphasized, however, that the presence of a lateralized ERP effect only supports the relatively weak inference that some component process is lateralized (see, e.g. [10]). The "obvious" lateralization, however, needs not be the correct one; "paradoxical" lateralizations of effects that arise from generators on the medial aspect of a hemisphere are reasonably well documented (e.g. [28]). Indeed, the possibility of paradoxical lateralization cannot be easily dismissed in this case, since blood flow increases linked to the processing of SO structures has been observed not only in dorsolateral prefrontal regions, which are believed to subserve working memory functions, but also to the left anterior cingulate [6], a brain region believed to play an important role in the deployment of attentional resources among other functions [25]. Frontal and prefrontal activations have also been reported with verbal working memory tasks [26] and with non-verbal, visual working memory tasks (e.g. [19]).

Another aspect of our data is the appearance of large slow potential effect at other, less anterior scalp locations. To some extent, a similar pattern was seen in previous work with visual sentences [15] and word pairs [17]; negativities with a similar distribution but of shorter duration than these slow potentials were seen for moderate working memory loads by Ruchkin et al. [32]. More recently, Rösler et al. [30] have used ERPs to investigate the fan effect and have shown very robust left anterior negativities related to the active retrieval and evaluation of information in these paradigms; moreover, the size of this frontal negativity increases with the difficulty of the comparison being made. Thus, more work will have to be done with both visual and auditory sentences, however, before we can be certain exactly what aspect of the processes involved are indexed by these slow potentials.
Our analysis of slow potential effects contrasts with our discussion of "faster" activity that was evoked by the end of the relative clause itself. Here, one could see some limitations in recording ERPs elicited by free speech stimuli, even where the timing of the physical (acoustic) stimulus is controlled. Because a linguistically meaningful segmentation of the utterance is difficult to recover except when the boundaries happen to coincide with major acoustic boundaries or pauses, the temporal boundaries of the cognitive events relevant to auditory language processing are likewise difficult to define with precision. Indeed, it is still unclear, even from a strictly linguistic point of view, which features of spoken language are the most basic units in language processing, e.g., the phonemes, syllables, acoustic-phonetic sequences, or more complex but as yet undescribed phonological entities [9]. In any case, the auditory recovery cycle is long enough $(\approx 10 \mathrm{sec})$ so that naturally spoken mid-sentence words are unlikely to generate crisp sensory evoked potentials. But, our results suggest that even though it would be possible via computer editing to produce stimulus sentences whose temporal properties were precisely controlled to the millisecond, this effort might be superfluous for investigating sentence processing. The existence of robust slow potential effects in auditory sentence processing apparently obviates the need to time-lock averages to the exact onset of some physical change in signal intensity (which may be ill defined). Additional sources of information about the linguistic features actualy used in auditory language processing - which would therefore be the experimental events of interest may quite possibly be found through the application of more advanced spectrum analysis methods to EEG data (e.g. [1,27]), whether that EEG was elicited by words spoken in isolation [38] or by words appearing in sentences [23]. Such developments promise to dampen further the desire to create experimentally the kinds of artificial "events" that are foreign to the natural speech stream, and apparently unnecessary for natural language understanding to take place.

\section{Acknowledgements}

Work reported in this paper was supported by DFG Grant Mu 797/2 to Horst M. Müller, and NICHD Grant HD22614 and by NIA Grant AG08313 to Marta Kutas. Jonathan W. King was also supported in part by a McDonnell-Pew postdoctoral fellowship from the San Diego Center for Cognitive Neuroscience, funds from the Center for Human Information Processing (CHIP; NIMH Grant MH14268), and funds from the Center for Research on Language (CRL; NIH Grant T32 DC00041-01) at UCSD. We would also like to thank Jill Weckerly for her comments on this work. 


\section{References}

[1] Başar, E. and Bullock, T.H. (Eds.), Brain Dynamics: Progress and Perspectives, Springer, Berlin, 1989, 547 pp.

[2] Besson, M., Faita, F., Czternasty, C. and Kutas, M., Event-related potential analyses of temporal disruptions in written and spoken sentences, Biol. Psychol., (1996) in press.

[3] Connolly, J.F., Phillips, N.A., Stewart, S.H. and Brake, W.G., Event-related potential sensitivity to acoustic and semantic properties of terminal words in sentences, Brain Lang., 43 (1992) 1-18.

[4] Friederici, A.D., Pfeifer, E. and Hahne, A., Event-related brain potentials during natural speech processing: effects of semantic, morphological and syntactic violations, Cogn. Brain Res., 1 (1993) 183-192.

[5] Ford, M., A method of obtaining measures of local parsing complexity throughout sentences, J. Verbal Learn. Verbal Behav., 22 (1983) 203-218.

[6] Grossman, M., Crino, P., Reivich, M., Stern, M.B. and Hurtig, H.I., Attention and sentence processing deficits in Parkinson's disease: the role of anterior cingulate cortex, Cereb. Cortex, 2 (1992) 513525 .

[7] Holcomb, P.J. and Neville, H.J., Natural speech processing: an analysis using event-related brain potentials, Psychobiology, 19 (1991) 286-300.

[8] Holmes, V.M. and O'Regan, J.K., Eye fixation patterns during the reading of relative-clause sentences, J. Verbal Learn. Verbal Behav., 20 (1981) 417-430.

[9] Kent, R.D., Auditory processing of speech. In: J. Katz, N.A. Stecker and D. Henderson (Eds.), Central Auditory Processing: a Transdisciplinary View, Mosby, St. Louis, MO, 1992, pp. 93-103.

[10] King, J.W., Ganis, G. and Kutas, M., Potential asymmetries in language comprehension: in search of the electrical right. In: M. Beeman and C. Chiarello (Eds.), Getting it Right: the Cognitive Neuroscience of Right Hemisphere Language Comprehension, Erlbaum, Amsterdam, 1996, in press.

[11] King, J. and Just, M.A., Individual differences in syntactic processing: the role of working memory, J. Mem. Lang., 30 (1991) 580-602.

[12] King, J.W. and Kutas, M., Who did what and when? Using wordand clause-related ERPs to monitor working memory usage in reading, J. Cogn. Neurosci., 7 (1995) 378-397.

[13] Kluender, R. and Kutas, M., Bridging the gap: evidence from ERPs on the processing of unbounded dependencies, J. Cogn. Neurosci., 5 (1993) 196-214.

[14] Kluender, R. and Kutas, M., Subjacency as a processing phenomenon, Lang. Cogn. Process., 8 (1993) 573-633.

[15] Kutas, M. and King, J.W., The potentials for basic sentence processing: differentiating integrative processes. In: I. Ikeda and J.L. McClelland (Eds.), Attention and Performance, Vol. XVI, MIT Press, Cambridge, MA, 1996, pp. 501-546.

[16] Kutas, M. and Van Petten, C., Psycholinguistics electrified: event-related brain potential investigations. In: M.A. Gernsbacher (Ed.), Handbook of Psycholinguistics, Academic Press, San Diego, CA, 1994, pp. 83-143.

[17] Lang, M., Lang, W., Uhl, F., Kornhuber, A., Deecke, L. and Kornhuber, H.H., Slow negative potential shifts indicating verbal cognitive learning in a concept formation task, Hum. Neurobiol., 6 (1987) 183-190.

[18] MacWhinney, B. and Pleh, C., The processing of restrictive relative clauses in Hungarian, Cognition, 29 (1988) 95-141.

[19] McCarthy, G., Blamire, A.M., Puce, A., Nobre, A.C., Bloch, G., Hyder, F., Goldman-Rakic, P. and Shulman, R.G., Functional magnetic resonance imaging of human prefrontal cortex activation during a spatial working memory task, Proc. Natl. Acad. Sci. (USA), 91 (1994) 8690-8694.
[20] Mecklinger, A., Schriefers, H., Steinhauer, K. and Friederici, A.D., Processing relative clauses varying on syntactic and semantic dimensions: an analysis with event-related potentials, Mem. Cogn., 23 (1995) 477-494.

[21] Miller, G.A. and Chomsky, N., Finitary models of language users. In: D. Luce, R. Bush and E. Galanter (Eds.), Handbook of Mathematical Psychology, Wiley, New York, 1963, pp. 419-492.

[22] Müller, H.M., Sprache und Evolution: Grundlagen der Evolution und Ansätze einer evolutionstheoretischen Sprachwissenschaft, De Gruyter, Berlin, 1990, 317 pp.

[23] Müller, H.M., Weiss, S. and Rappelsberger, P., EEG coherence analysis of auditory sentence processing. In: H. Witte and $\mathrm{U}$. Zwiener (Eds.), Quantitative and Topological EEG and MEG Analysis, Universitätsverlag Jena, Jena, in press.

[24] Osterhout, L. and Holcomb, P.J., Event-related potentials and syntactic anomaly: evidence of anomaly detection during the perception of continuous speech, Lang. Cogn. Process., 8 (1993) 413-437.

[25] Petrides, M., Alivisatos, B., Meyer, E. and Evans, A.C., Functional activation of the human frontal cortex during performance of verbal working memory tasks, Proc. Natl. Acad. Sci. (USA), 90 (1993) 878-882.

[26] Posner, M.I. and Petersen, S.E., The attention system of the human brain, Annu. Rev. Neurosci., 13 (1990) 25-42.

[27] Rappelsberger, P., Lacroix, D. and Petsche, H., Amplitude and coherence mapping: its application in psycho- and patho-physiological studies. In: M. Rother and U. Zwiener (Eds.), Quantitative EEG Analysis: Clinical Utility and New Methods, Universitätsverlag, Jena, 1993, pp. 179-186.

[28] Regan, D., Human Brain Electrophysiology: Evoked Potentials and Evoked Magnetic Fields in Science and Medicine, Elsevier, New York, 1989, 672 pp.

[29] Rockstroh, B., Elbert, T., Canavan, A., Lutzenberger, W. and Birbaumer, N., Slow Cortical Potentials and Behavior, Urban and Schwarzenberg, Baltimore, MD, 1989, 267 pp.

[30] Rösler, F., Heil, M. and Glowalla, U., Monitoring retrieval from long-term memory by slow event-related brain potentials, Psychophysiology, 30 (1993) 170-182.

[31] Rösler, F., Heil, M. and Hennighausen, E., Exploring memory functions by means of brain electrical topography: a review, Brain Topogr., 7 (1995) 301-313.

[32] Ruchkin, D.S., Johnson, R., Canoune, H. and Ritter, W., Short-term memory storage and retention: an event-related brain potential study, Electroenceph. Clin. Neurophysiol., 76 (1990) 419-439.

[33] Schriefers, H., Friederici, A.D. and Kuhn, K., The processing of locally ambiguous relative clauses in German, J. Mem. Lang., 34 (1995) 499-520.

[34] Sheldon, A., The role of parallel function in the acquisition of relative clauses in English, J. Verbal Learn. Verbal Behav., 13 (1974) 272-281.

[35] Shipley-Brown, F., Dingwall, W.O. and Berlin, C.I., Hemispheric processing of affective and linguistic intonation contours in normal subjects, Brain Lang., 33 (1988) 16-26.

[36] Wanner, E. and Maratsos, M., An ATN approach to comprehension. In: M. Halle, J. Bresnan and G.A. Miller (Eds.), Linguistic Theory and Psychological Reality, MIT Press, Cambridge, MA, 1978, pp. 119-160.

[37] Weckerly, J. and Kutas, M., The interaction of noun animacy and grammatical role in the processing of object relative sentences, Submitted for publication.

[38] Weiss, S. and Rappelsberger, P., EEG coherences within the 13-18 $\mathrm{Hz}$ band as correlates of a distinct lexical organization of concrete and abstract nouns in humans, Neurosci. Lett., 209 (1996) 17-20.

[39] Woodward, S.H., Ford, J.M. and Hammett, S.C., N4 to spoken sentences in young and older subjects, Electroenceph. Clin. Neurophysiol., 87 (1993) 306-320. 